\title{
Young adults' physical activity and physical function: 12 months follow up
}

\section{BRUNO SILVA ${ }^{1,2 *}$ | PEDRO BEZERRA ${ }^{1,2}$ | JOSÉ M. CANCELA ${ }^{3}$}

1 Escola Superior de Desporto e Lazer, Instituto Politécnico de Viana do Castelo, Melgaço, Portugal.

${ }^{2}$ Research Center in Sports Science, Health and Human Development (CIDESD), Vila Real, Portugal.

${ }^{3}$ Faculty of Education and Sports Science, University of Vigo, Pontevedra, Spain.

Correspondence to: Escola Superior de Desporto e Lazer, Portugal, Complexo Desportivo e Lazer Comendador Rui Solheiro, Monte de Prado, Zip Code 4960-320,

Melgaço, Portugal. Tel.: +351-258-809-678.

email: silvabruno@esdl.ipvc.pt

https://doi.org/10.20338/bjmb.v15i2.200

\section{HIGHLIGHTS}

- After 12 months, young adults' physical function accessed by the Functional Movement Screen changes in accordance with gender and habitual PA.

- Functional Movement Screen may be sensitive to improvements or decrements due to contextual environments that over-or underproduce the stimulation required to develop those skills.

- Sports Science university students' daily routines may be interceding physical activity patterns and consequently PFn.

- After 12 months, Sports Science men students improve more sports-specific physical function skills.

- After 12 months, Sports Science women students improve total physical function and core stability tasks.

\section{ABBREVIATIONS}

ASLR Active straight-leg raise

DS Deep squat

EF Effect size

FMS Functional Movement Screen

HS Hurdle step

ILL In-line lunge

PA Physical activity

PFn Physical function

RS Rotary stability

SM Shoulder mobility

TSP Trunk stability push-up

\section{PUBLICATION DATA}

Received 26092020

Accepted 06012021

Published 01062021
BACKGROUND: The knowledge about university students generally demonstrates that they do not meet current recommendations for Physical Activity (PA). However, PA and Physical Fitness are not an indicator for Physical Function (PFn), being PFn a separate construct that is related to movement skill proficiency.

AIM: To analyze the rate of changes in PFn accessed by the Functional Movement Screen (FMS) and PA levels (sports and leisure) in a group of Sport Science university students after one year.

METHOD: 41 men (22.3 \pm 5.7 years) and 22 women (20.7 $\pm 0.9 y e a r s)$ participated in this research. The data were collected in the first month of the academic year and after 12 months in the following order: Baecke Habitual Physical Activity Questionnaire, anthropometrics, and FMS.

RESULTS: PFn assessment, from baseline to second assessment, revealed significant changes in Trunk Stability Push-up ( $p=0.003$; $E S=0.656$ ) and total score $(p=0.026$; $E S=0.497)$ for women and in Deep Squat $(p=0.003$; $E S=0.334$ ) for men. No significant changes were found in women PA levels. Men decreased in leisure PA $(p=0.002 ; E S=0.538)$ and total PA ( $p=0.003 ; E S=0.519)$.

CONCLUSION: Sports Science university students' daily routines may mediate physical activity patterns with following physical function improvements or decrements, occurring due to contextual environments that over-or under-produce the stimulation required to develop those skills.

KEYWORDS: Functional fitness | University students | Physical fitness | Functional Movement Screen

\section{INTRODUCTION}

The literature demonstrates that university students do not meet current recommendations for physical activity (PA) levels ${ }^{1}$ and are less active than children and adolescents. ${ }^{2}$ The transition from high school to college involves the development of individual habits. During this transition, students undergo emotional, physiological, and 
environmental changes that influence several behaviors (e.g., consumer habits and lifestyle aspects, such as PA and weight status). ${ }^{3}$

Generally, studies from different cultures and countries demonstrate that female students tend to be more sedentary and perform less PA than male students. ${ }^{1,4} \mathrm{~A}$ recent study compared university students from the Vocational School of Health Services, the School of Physical Therapy and Rehabilitation, the Faculty of Nursing, the School of Sports Sciences and Technology, and the Faculty of Medicine. The study revealed that students from the School of Sports Sciences performed more PA than other students. ${ }^{4}$ This factor was mediated by being involved in PA in earlier years and the fact that these students' family members also tended to be physically active. According to other schools, these students were considered a reference point for determining physical inactivity status. ${ }^{4}$ Being involved in PA practice early in life and continuing this involvement as a lifestyle choice can influence one's involvement in PA during later years. ${ }^{5}$ Moreover, university students who engage in PA use on-campus sports facilities-one of the most often cited reasons for physical inactivity is inadequate on-campus sports facilities. ${ }^{4} \mathrm{~A}$ few studies have analyzed PA patterns in university students in Portugal. ${ }^{1}$ However, recently research that includes very active university students demonstrated that similar than in children and adolescents, the levels of motor competence positively influence weight status. ${ }^{6}$

For practical reasons, most studies use questionnaires to assess PA. ${ }^{7}$ In general, these questionnaires are designed to minimize potential confounding effects. However, there is no consensus on which questionnaire is the best among the several validated and widely used but the need to choose the questionnaire that best suits the objectives. ${ }^{8}$

However, this information is not an indicator of physical function (PFn) related to movement skill proficiency. ${ }^{9}$

PFn and physical fitness are separate constructs. ${ }^{10} \mathrm{PFn}$ is more closely associated with functional movement, which relates to the body's multi-planar and multi-joint movements (specifically, simultaneous mobility and stability) and optimal movement. ${ }^{11}$ Optimal movement can be defined for all age ranges and trends to refine coordination, strength, and endurance, concluding in improvements to daily living activities, physical wellbeing and/or sports performance. ${ }^{12}$

Gray Cook et al. ${ }^{13}$ developed the Functional Movement Screen (FMS) to perform pre-participation functional evaluations. This screening tool comprises a battery of tests to evaluate joint mobility and stability simultaneously based on a series of seven movements. ${ }^{13}$ Although none of the tests was designed with a specific sport in mind, they challenge the upper and lower extremities and the trunk to establish which physical or functional limitation is the most significant. ${ }^{13}$

The current knowledge about the relationship between FMS and PA is limited. Some research demonstrate that FMS scores can change during a soccer or volleyball season among collegiate players. ${ }^{14}$ However, recent studies demonstrate that FMS is only linked to motor competence stability and not to all motor competence constructs (locomotor, manipulative, and stability) ${ }^{15}$,thus, might be suitable for determining PFn but not for discriminating performance. ${ }^{16}$ This is because it has been linked to only some sport-specific performance tasks. ${ }^{17}$ Compared to men, women have lower mean motor competence values and perform differently in some FMS tasks..$^{15}$ Men usually yield higher scores in the FMS 
trunk stability push-up test, while women perform better in the FMS active straight leg raise. 15

Therefore, this research aimed to analyze the rate of changes in PFn accessed by FMS and PA levels (sports and leisure) in a group of sports science students after one year.

\section{METHODS}

\section{Participants}

All participants were volunteers, consisting of 41 men $(22.3 \pm 5.7$ years; $74.0 \pm 1.4 \mathrm{~kg}$; $176.7 \pm 0.9$ meters) and 22 women (20.7 \pm 0.9 years; $59.4 \pm 1.7 \mathrm{~kg}$; $162.5 \pm 1.1$ meters). Participants had no motor, cognitive, or health impairments that could affect their performance. The criteria of inclusion were: (i) be enrolled and attending the Sports and Leisure degree; (ii) participate in both assessments (before and after one-year); (iii) be enrolled in more than $60 \%$ of the sports applied disciplines; (iv) injury-free at the time of the evaluation (injury is understood as any complaint of physical or psychological parameters that result in instability to practice or normally complete in a particular sport or physical activity); (v) at the end of the academic year do not fail any practical disciplines due to absenteeism.

After being briefed about the study design and potential risks and benefits of their participation, participants signed a free informed consent following the ethical standards for the study in humans as suggested by the Declaration of Helsinki. The study was approved by the Scientific Council (CTC-ESDL-CE002-2017).

\section{Procedures}

All participants had their anthropometrics, habitual physical activity, and physical function assessed. Firstly, to characterize the Physical Activity profile, all subjects fulfilled the Habitual Physical Activity Questionnaire validated for the Portuguese Population ${ }^{18}$ and gave their informed consent. During the morning, the assessments were made in an indoor facility with groups of around 20 participants at an average temperature of $26^{\circ} \mathrm{C}$ and relative humidity of $18^{\circ}$. The data were collected in the biomechanics laboratory in the first month of the academic year (from October to November) and repeated after 12 months. The tests were conducted in the following sequence: Physical Activity questionnaire; anthropometric and physical function.

\section{Habitual Physical Activity Questionnaire}

The Portuguese version of the Baecke Habitual Physical Activity Questionnaire 18 was applied. This 12 months retrospective questionnaire comprehend habitual physical activity scores that includes eight items grouped in two dimensions: 1- Physical Activity Sports (4 items) - evaluate the physical activity performed in the sport or programmed physical exercise practiced during leisure hours and 2- Physical Activity - Leisure (4 items) - evaluate physical activity in activities other than sport, practiced during leisure time (e.g., walking, cycling). The answers are scored on a five-point scale, except for the sports question, with a higher score for each item corresponding to higher physical activity. Each of the two groups or dimensions results in a partial index of physical activity (Sports and Leisure). The total physical activity was calculated by the sum of the two partial values.

\begin{tabular}{l|l|l|l|ll}
\hline $\begin{array}{l}\text { Silva, Bezerra, } \\
\text { Cancela }\end{array}$ & 2021 & VOL.15 & N.2 & https://doi.org/10.20338/bjmb.v15i2.200
\end{tabular}




\section{Anthropometric}

During the evaluations, all participants stood barefoot and dressed in light clothing with the heights measured through a portable stadiometer (SECA 217, Germany) and body mass with a scale (SECA 760, Germany). Height measurements were rounded to the nearest $0.1 \mathrm{~cm}$, with the head oriented according to the Frankfurt plane and body mass to the nearest $0.5 \mathrm{~kg}$.

\section{Physical Function}

Physical function was measured following the battery developed by Gray Cook and associates. ${ }^{13}$ The Functional Movement Screen (FMS) is a screening tool that simplifies the assessment of fundamental movement pattern $\mathbf{s}^{13}$ according to seven movements - deep squat (DS), hurdle step (HS), in-line lunge (ILL), shoulder mobility (SM), active straight-leg raise (ASLR), trunk stability push-up (TSP), and rotary stability (RS) - and three clearing examinations. The clearing examinations (shoulder clearing test, spinal extension clearing test, and spinal flexion clearing test) were not scored. However, they were performed to determine whether the participant was able to perform the assessments. Three attempts of each pattern were completed, and the best-performed repetition was scored on a scale of 0 to 3 as follows: 0 = pain anywhere in the body; $1=$ unable to complete the movement pattern or unable to assume the position to perform the movement, $2=$ able to complete the movement but must compensate in some way to perform the fundamental movement, $3=$ able to perform the movement correctly without any compensation, complying with standard movement expectations associated with each test. ${ }^{13}$

A certified FMS specialist with four years of experience conducted the tests according to the standard protoco ${ }^{13}$ with an official FMS test kit. Approximately 10 seconds of rest were provided between trials, and one minute of rest was allowed between tests. Except for the DS and TSP, each side of the body was assessed unilaterally with the best scores for each of the seven tests registered for analysis and used to calculate a composite score.

These assessment protocols' reliability has been established with moderate to excellent levels of agreement in trained raters. ${ }^{13}$ For each FMS test, all participants were assigned to one of the three groups (low, medium, and high limit) according to the archived score $(0$ or 1 = low, 2 = medium, 3 = high).

\section{Statistical Analyses}

Averages and $95 \%$ confidence intervals for lower and upper limits were calculated according to descriptive statistics. After applying the Shapiro-Wilk test in conjunction with a histogram, it was verified that the sample distribution was not normal, and the Wilcoxon signed-rank test was applied. The effect size (ES) to non-parametric tests was obtained ${ }^{19}$ : $r=\frac{|z|}{\sqrt{N}}$, where $N$ is the total sample size, and the value of $z$ is reported after applying the Wilcoxon signed-rank test. The classification of ES was obtained by using the following criteria (19): very small effect $(r<0.1)$; small effect $(0.1 \leq r<0.3)$; medium effect $(0.3 \leq r<$ 
$0.5)$; and large effect $(r \geq 0.5)$. The analyses were performed separated by gender since women tend to be less physically active than men ${ }^{1,4}$ and perform differently in specific FMS tasks. ${ }^{15}$

Percentages of difference were calculated using a Microsoft Excel spreadsheet by determining the coefficient of variation (by dividing the standard deviation by the mean and multiplying the result by one hundred) of each variable and then subtracting the first assessment values and the second assessment. The use of the variation coefficient allows the observation of the extent of variation concerning the mean, thus increasing the precision of comparisons of different values measures. The remaining statistical analyses were done using the Statistical Package for Social Sciences (SPSS, Version 25.0, Chicago, IL) with the significance level set at $p<0.05$.

\section{RESULTS}

A drop out of $4.5 \%$ for women in the FMS assessment and $19.5 \%$ in the anthropometric and PA assessment for men was verified.

Significant improvements between the first and second assessments of women were found in FMS TSP $(p=0.003)$ and FMS total score $(p=0.026)$ (Table 1).

Table 1 - Differences between the first and second physical function assessments for women.

\begin{tabular}{|c|c|c|c|c|}
\hline $\mathrm{N}=21$ & $\begin{array}{c}1^{\text {st }} \text { Assessment } \\
\text { Mean (SD) } \\
{[95 \% \mathrm{Cl}]}\end{array}$ & $\begin{array}{c}2^{\text {2nd }} \text { Assessment } \\
\text { Mean (SD) } \\
{[95 \% \mathrm{Cl}]}\end{array}$ & $\begin{array}{l}\text { Percentage } \\
\text { difference }\end{array}$ & $\begin{array}{l}\text { Effect } \\
\text { size }\end{array}$ \\
\hline FMS Squat & $\begin{array}{c}2.1(0.2) \\
{[1.7-2.4]}\end{array}$ & $\begin{array}{c}2.2(0.1) \\
{[2.0-2.4]}\end{array}$ & $5.0 \%$ & 0.178 \\
\hline FMS Hurdle Step & $\begin{array}{c}1.6(0.1) \\
{[1.3-1.9]}\end{array}$ & $\begin{array}{c}1.9(0.1) \\
{[1.7-2.2]}\end{array}$ & $1.0 \%$ & 0.350 \\
\hline FMS In Line Lunge & $\begin{array}{c}2.1(0.2) \\
{[1.8-2.4]}\end{array}$ & $\begin{array}{c}2.2(0.1) \\
{[2.0-2.4]}\end{array}$ & $5.0 \%$ & 0.126 \\
\hline FMS Shoulder Mobility & $\begin{array}{c}2.1(0.2) \\
{[1.7-2.5]}\end{array}$ & $\begin{array}{c}2.2(0.2) \\
{[1.8-2.5]}\end{array}$ & $0.4 \%$ & 0.126 \\
\hline FMS Straight Leg Raise & $\begin{array}{c}2.8(0.1) \\
{[2.6-3.0]}\end{array}$ & $\begin{array}{c}2.6(0.1) \\
{[2.4-2.9]}\end{array}$ & $-0.3 \%$ & 0.178 \\
\hline FMS Rotary Stability & $\begin{array}{c}2.0(0.0) \\
{[2.0-2.0]}\end{array}$ & $\begin{array}{c}1.9(0.1) \\
{[1.7-2.1]}\end{array}$ & $-5.3 \%$ & 0.218 \\
\hline FMS Trunk Stability Push-up & $\begin{array}{c}(0.1) \\
{[0.8-1.3]}\end{array}$ & $\begin{array}{c}1.9(0.1)^{*} \\
{[1.3-2.5]}\end{array}$ & $14.7 \%$ & 0.656 \\
\hline FMS total score & $\begin{array}{c}13.8(0.5) \\
{[12.7-14.8]}\end{array}$ & $\begin{array}{c}15.0(0.6)^{*} \\
{[13.8-16.1]}\end{array}$ & $0.4 \%$ & 0.497 \\
\hline
\end{tabular}

FMS - Functional Movement Screen; ${ }^{*}$ significant differences, $p<0.05$;

Significant improvements between assessments were found on FMS DS $(p=0.003)$ in men (Table 2). 
Table 2 - Differences between the first and second physical function assessments for men.

\begin{tabular}{|c|c|c|c|c|}
\hline $\mathrm{N}=41$ & $\begin{array}{c}1 \text { st Assessment } \\
\text { Mean (SD) } \\
{[95 \% \mathrm{Cl}]}\end{array}$ & $\begin{array}{c}2^{\text {2nd }} \text { Assessment } \\
\text { Mean (SD) } \\
{[95 \% \mathrm{Cl}]}\end{array}$ & $\begin{array}{l}\text { Percentage } \\
\text { difference }\end{array}$ & $\begin{array}{c}\text { Effect } \\
\text { size }\end{array}$ \\
\hline \multirow{2}{*}{ FMS Squat } & $2.0(0.1)$ & $2.2(0.1)^{*}$ & \multirow{2}{*}{$0.5 \%$} & \multirow{2}{*}{0.334} \\
\hline & {$[1.8-2.2]$} & {$[2.1-2.4]$} & & \\
\hline \multirow{2}{*}{ FMS Hurdle Step } & $1.7(0.1)$ & $1.9(0.1)$ & \multirow{2}{*}{$0.6 \%$} & \multirow[t]{2}{*}{0.276} \\
\hline & {$[1.5-1.9]$} & {$[1.7-2.0]$} & & \\
\hline \multirow{2}{*}{ FMS In Line Lunge } & $(0.1)$ & $1.9(0.1)$ & \multirow{2}{*}{$-0.3 \%$} & \multirow{2}{*}{0.089} \\
\hline & {$[1.9-2.0]$} & {$[1.8-2.0]$} & & \\
\hline \multirow{2}{*}{ FMS Shoulder Mobility } & $1.9(0.1)$ & $(0.1)$ & \multirow{2}{*}{$0.3 \%$} & \multirow[t]{2}{*}{0.166} \\
\hline & {$[1.6-2.1]$} & {$[1.7-2.3]$} & & \\
\hline \multirow{2}{*}{ FMS Straight Leg Raise } & $2.2(0.1)$ & $2.3(0.1)$ & \multirow{2}{*}{$0.2 \%$} & \multirow{2}{*}{0.187} \\
\hline & {$[2.2-2.6]$} & {$[2.1-2.4]$} & & \\
\hline \multirow{2}{*}{ FMS Rotary Stability } & $2.0(0.1)$ & $2.0(0.1)$ & \multirow{2}{*}{$0.0 \%$} & \multirow{2}{*}{0.154} \\
\hline & {$[1.9-2.0]$} & {$[1.9-2.1]$} & & \\
\hline \multirow{2}{*}{ FMS Trunk Stability Push-up } & $2.4(0.1)$ & $2.5(0.1)$ & \multirow{2}{*}{$0.2 \%$} & \multirow{2}{*}{0.180} \\
\hline & {$[2.1-2.6]$} & {$[2.2-2.8]$} & & \\
\hline \multirow{2}{*}{ FMS total score } & $14.2(0.3)$ & $14.7(0.3)$ & \multirow{2}{*}{$0.1 \%$} & \multirow{2}{*}{0.233} \\
\hline & {$[13.5-14.9]$} & {$[14.2-15.3]$} & & \\
\hline
\end{tabular}

FMS - Functional Movement Screen; ${ }^{*}$ significant differences, $p<0.05$;

Women significantly increased the body mass $(p=0.000)$. Descriptive statistics of anthropometric conducted on women can be found in Table 3. No significant changes in PA levels were found in women.

Table 3 - Differences between the first and second anthropometric and physical activity levels assessment for women.

\begin{tabular}{|c|c|c|c|c|}
\hline $\mathrm{N}=22$ & $\begin{array}{c}1^{\text {st }} \text { Assessment } \\
\text { Mean (SD) } \\
{[95 \% \mathrm{Cl}]}\end{array}$ & $\begin{array}{c}2^{\text {nd }} \text { Assessment } \\
\text { Mean (SD) } \\
{[95 \% \mathrm{Cl}]}\end{array}$ & $\begin{array}{l}\text { Percentage } \\
\text { difference }\end{array}$ & Effect size \\
\hline Body mass (kg) & $\begin{array}{c}57.5(1.7) \\
{[54.2-61.1]}\end{array}$ & $\begin{array}{c}59.4(1.7)^{*} \\
{[55.8-63.1]}\end{array}$ & $0.1 \%$ & 0.734 \\
\hline Height (cm) & $\begin{array}{c}162.3(1.2) \\
{[159.9-164.7]}\end{array}$ & $\begin{array}{c}162.5(1.1) \\
{[160.2-164.9]}\end{array}$ & $0.1 \%$ & 0.245 \\
\hline PAQ - Sport & $\begin{array}{c}4.1(0.3) \\
{[3.5-4.6]}\end{array}$ & $\begin{array}{c}(0.2) \\
{[3.5-4.5]}\end{array}$ & $-2.3 \%$ & 0.400 \\
\hline PAQ - Leisure & $\begin{array}{c}4.3(0.1) \\
{[4.1-4.6]}\end{array}$ & $\begin{array}{c}4.1(0.3) \\
{[3.5-4.7]}\end{array}$ & $-5.0 \%$ & 0.800 \\
\hline PAQ - Total & $\begin{array}{c}8.3(0.3) \\
{[7.8-9.0]}\end{array}$ & $\begin{array}{c}8.1(0.4) \\
{[7.3-8.9]}\end{array}$ & $-1.4 \%$ & 0.800 \\
\hline
\end{tabular}

PAQ - Physical Activity Questionnaire; $\mathrm{cm}$ - centimetres; kg - kilograms; \% - percent; g/cm² - grams per square centimetre; * significant differences, $p<0.05$;

Table 4 presents the descriptive statistics of anthropometric and PA levels of men in both assessments. It was also observed a significant decrease in leisure PA score at 0.8 points $(p=0.002)$ and total PA score at 1.1 points $(p=0.003)$. 
Table 4 - Differences between the first and second anthropometric and physical activity levels for men.

\begin{tabular}{|c|c|c|c|c|}
\hline$N=33$ & $\begin{array}{c}\text { st Assessment } \\
\text { Mean (SD) } \\
{[95 \% \mathrm{Cl}]}\end{array}$ & $\begin{array}{c}2^{\text {nd }} \text { Assessment } \\
\text { Mean (SD) } \\
{[95 \% \mathrm{Cl}]}\end{array}$ & $\begin{array}{l}\text { Percentage } \\
\text { difference }\end{array}$ & $\begin{array}{l}\text { Effect } \\
\text { size }\end{array}$ \\
\hline Body mass $(\mathrm{kg})$ & $\begin{array}{c}73.8(1.3) \\
{[71.1-76.5]}\end{array}$ & $\begin{array}{c}74.0(1.4) \\
{[71.2-76.8]}\end{array}$ & $0.1 \%$ & 0.083 \\
\hline Height (cm) & $\begin{array}{c}176.3(0.8) \\
{[174.6-177.9]}\end{array}$ & $\begin{array}{c}176.7(0.9)^{*} \\
{[175.0-178.4]}\end{array}$ & $0.1 \%$ & 0.376 \\
\hline PAQ - Sport & $\begin{array}{c}4.0(0.2) \\
{[3.6-4.3]} \\
\end{array}$ & $\begin{array}{c}3.6(0.2) \\
{[3.6-4.3]} \\
\end{array}$ & $-0.6 \%$ & 0.274 \\
\hline PAQ - Leisure & $\begin{array}{c}4.4(1,2)^{*} \\
{[4.0-4.7]}\end{array}$ & $\begin{array}{c}3.6(0.2) \\
{[3.2-3.9]}\end{array}$ & $-21.7 \%$ & 0.495 \\
\hline PAQ - Total & $\begin{array}{c}8.3(0.3)^{*} \\
{[7.6-8.9]}\end{array}$ & $\begin{array}{c}7.2(0.3) \\
{[6.7-7.7]}\end{array}$ & $-0.6 \%$ & 0.477 \\
\hline
\end{tabular}

PAQ - Physical Activity Questionnaire; $\mathrm{cm}$ - centimetres; $\mathrm{kg}$ - kilograms; $\mathrm{g} / \mathrm{cm}^{2}$ - grams per square centimetre; " significant differences, $p<0.05$.

\section{DISCUSSION}

This research provides an insight into the rate of changes in PFn accessed by FMS and PA levels in a group of sports science students for one year. The results suggest that some specific aspects of PFn accessed by FMS are sensitive to improvements or decreases due to contextual environments that over-or under-produce the stimulation required to develop specific skills. It seems that young adults' PFn accessed by the Functional Movement Screen presents different trajectories based on sex and PA practice.

After 12 months, no significant changes were seen in women's PA levels, whereas men's leisure and total PA decreased. The daily routines associated with university life (e.g., time management, academic activities, leisure activities, and motivation) ${ }^{20}$ might be responsible for the significant decreases observed in mean total PA $(-0.6 \%)$ and leisure PA $(-21.7 \%)$. Thus, such routines might influence physical fitness and other physical health outcomes. $^{21}$

Humans are motivated by their fundamental psychological needs for competence, autonomy, and relatedness. ${ }^{22}$ Compared with the normative for the FMS total score, ${ }^{23}$ men and women present lower baseline and average scores. However, after 12 months, men significantly improved FMS DS, and women improved FMS TSP and FMS total scores. These results are in line with other studies that have reported that specific movements can change during a season in soccer and among collegiate volleyball players. ${ }^{14}$ When observing the amount of PA practice, we can assume that PA habits were different between the study groups throughout these 12 months. However, we have not analyzed the differences statistically.

Men's PA practice decreased substantially, but their performance improved regarding movements that challenge total body mechanics, which are needed in most athletic events. ${ }^{13}$ This change could be related to the fact that PFn and physical 
performances are separate constructs ${ }^{10}$. Engaging in PA is not the only factor that positively influences PFn and the various physical fitness components. Even when total PA was not improved, during this period, all individuals were required to practice soccer, volleyball, handball, roller hockey, athletic running, throwing, and jumping events, and rock climbing. This variety of physical demands led to new adaptations and improvements in general motor proficiency. ${ }^{24}$ Such outcomes were more evident in women than in men since women tend to be less physical active ${ }^{1,4}$ and possess fewer motor competence ${ }^{15}$ than men. These aspects and the significant height growth (verified only in men) might also lead to new adaptations and changes in PFn. Indeed, women tend to produce lower scores in the FMS TSP than men, ${ }^{11}$ but the present improvement was based on a comparison with mean values at baseline. This comparison indicates low mean scores and normal scores after 12 months. The FMS TSP in women has been described as more sensitive to core stability than upper extremity strength. ${ }^{25}$ When considered separately of other factors, FMS TSP can be a reliable measure of PFn in specific populations. ${ }^{10}$ This improvement might be directly linked to new and/or more challenging motor experiences related to the implication of curriculum practices that demand different sports/skills expertise. The FMS total score directly depends on the remaining scores, ${ }^{13}$ and a significant improvement was mediated by a one-point improvement in the FMS TSP mean value.

As mentioned, this study showed that men's PA significantly decreased and more sports-specific PFn skills improved. Women maintained about the same level of PA practice while improving total PFn and core stability. However, further inquiry is necessary to determine how university students' time management, academic activities, leisure activities, and study time influence PFn and healthy habits, such as PA routines. When promoting PA among college students, it might be worth considering the role of sex differences and the extent to which PFn is accessed by FMS. These factors are common fundamental aspects of the human movement that can be changed based on the contextual environment. As such, they can either over-or under-produce the stimulation required to develop those skills.

This study has other potential limitations aside from that concerning sample size. Namely, the distribution between groups and the changes in each group's fundamental movement might have been influenced by other formal strength and conditioning training practices or a lack of training. Future research should clarify the role of the interaction between FMS and physical fitness, consider the influence of daily university routines, and the fact that a single year may not provide a broad enough framework considering the age group and the lack of studies addressing PFn in this population.

\section{CONCLUSION}

After 12 months, young adults' physical function presents different trajectories in accordance with gender, suggesting that some specific aspects of physical function accessed by the Functional Movement Screen may be sensitive to improvements or decrements due to contextual environments that over-or under-produce the stimulation required to develop those skills.

Sports Science university students' daily routines may be interceding physical activity patterns with men physical activity practice significantly decrease, and more sports 
specific physical function skills improve. Women maintaining about the same physical activity practice improve total physical function and core stability.

\section{REFERENCES}

1. Clemente FM, Nikolaidis PT, Martins FML, Mendes RS. Physical activity patterns in university students: Do they follow the public health guidelines? PLoS One. 2016. doi:10.1371/journal.pone.0152516

2. Cocca A, Liukkonen J, Mayorga-Vega D, Viciana-Ramírez J. Health-Related Physical Activity Levels in Spanish Youth and Young Adults. Percept Mot Skills. 2014. doi:10.2466/10.06.PMS.118k16w1

3. Gallardo-Escudero A, Muñoz Alférez MJ, Planells del Pozo EM, López Aliaga I. [The university stage does not favor the healthy life style in women students from Granada]. Nutr Hosp. 2014. doi:10.3305/nh.2015.31.2.8303

4. Dayi A, Acikgoz A, Guvendi G, Bayrak L, Ersoy B, Gur C, Ozmen O. Determination of Factors Affecting Physical Activity Status of University Students on a Health Sciences Campus. Med Sci Monit. 2017. doi:10.12659/MSM.899816

5. Bronikowski M, Bronikowska M, Pluta B, Maciaszek J, Tomczak M, Glapa A. Positive Impact on Physical Activity and Health Behaviour Changes of a 15-Week Family Focused Intervention Program: "juniors for Seniors." Biomed Res Int. 2016. doi:10.1155/2016/5489348

6. Silva B, Rodrigues LP, Clemente FM, Bezerra P, Cancela-Carral JM. Motor Competence and Body Composition in young adults: An exploratory study. Obes Med. 2019;14:100087. doi:https://doi.org/10.1016/j.obmed.2019.100087

7. Camões M, Severo M, Santos AC, Barros H, Lopes C. Testing an adaptation of the EPIC Physical Activity Questionnaire in Portuguese adults: A validation study that assesses the seasonal bias of self-report. Ann Hum Biol. 2010;37:185-197. doi:10.3109/03014460903341836

8. Dowd KP, Szeklicki R, Minetto MA, Murphy MH, Polito A, Ghigo E, van der Ploeg H, Ekelund U, Maciaszek J, Stemplewski R, et al. A systematic literature review of reviews on techniques for physical activity measurement in adults: A DEDIPAC study. Int J Behav Nutr Phys Act. 2018;15:15. doi:10.1186/s12966-017-0636-2

9. O'Brien W, Duncan M, Farmer O, Lester D. Do Irish Adolescents Have Adequate Functional Movement Skill and Confidence? J Mot Learn Dev. 2017. doi:10.1123/jmld.2016-0067

10. Silva B, Clemente FM, Lourenco Martins FM. Associations between Functional Movement Screen scores and performance variables in surf athletes. J Sports Med Phys Fitness. 2017. doi:10.23736/S0022-4707.17.07154-7 
11. Abraham A, Sannasi R, Nair R. Normative values for the functional movement screen in adolescent school aged children. Int J Sports Phys Ther. 2015;10:29-36.

12. Walter R. Thompson. Worldwide survey of fitness trends for 2019. ACSM's Heal Fit J. 2018. doi:10.1249/FIT.0000000000000438

13. Cook G, Burton L, Hoogenboom BJ, Voight M. Functional movement screening: the use of fundamental movements as an assessment of function - part 1. Int J Sports Phys Ther. 2014:9:396-409.

14. Mokha M, Sprague PA, Gatens DR. Predicting Musculoskeletal Injury in National Collegiate Athletic Association Division II Athletes From Asymmetries and Individual-Test Versus Composite Functional Movement Screen Scores. J Athl Train; 2016;51:276-282. doi:10.4085/1062-6050-51.2.07

15. Silva B, Rodrigues LP, Clemente FM, Cancela JM, Bezerra P. Association between motor competence and functional movement screen scores. PeerJ. 2019;2019: doi: $10.7717 /$ peerj. 7270

16. Silva B, Clemente FM, Camoes M, Bezerra P. Functional Movement Screen Scores and Physical Performance among Youth Elite Soccer Players. SPORTS (2017) 5: doi:10.3390/sports5010016

17. Silva B, Clemente FM, Martins FM. Associations between functional movement screen scores and performance variables in surf athletes. J Sports Med Phys Fitness. 2018;58: doi:10.23736/S0022-4707.17.07154-7

18. Almeida MCB, Ribeiro JLP. Adaptação do Habitual Physical Activity Questionnaire (Baecke), versão modificada, para a população portuguesa. Rev Enf Ref. 2014;serlV:2736. doi:10.12707/RIII1303

19. Pallant J. Spss Survival Manual: A Step by Step Guide to Data Analysis Using the SPSS Program. Australia: Allen \& Unwin. 2011.

20. Kondrič M, Sindik J, Furjan-Mandić G, Schiefler B. Participation motivation and student's physical activity among sport students in three countries. J Sport Sci Med. 2013;12:10-18.

21. Calestine J, Bopp M, Bopp CM, Papalia Z. College Student Work Habits are Related to Physical Activity and Fitness. Int J Exerc Sci. 2017;10:1009-1017.

22. Murphy JJ, MacDonncha C, Murphy MH, Murphy N, Nevill AM, Woods CB. What Psychosocial Factors Determine the Physical Activity Patterns of University Students? J Phys Act Health. 2019;16:325-332. doi:10.1123/jpah.2018-0205

23. Schneiders AG, Davidsson AH, Hörman E, Sullivan J. Functional movement screen normative values in a young, active population. Int J Sports Phys Ther. 2011,6:75-82.

24. Thelen E. Dynamic Systems Theory and the Complexity of Change. Psychoanal Dialogues Int J Relational Perspect. 2005;15:255-283. doi:10.1080/10481881509348831

25. Chorba RS, Chorba DJ, Bouillon LE, Overmyer CA, Landis JA. Use of a functional movement screening tool to determine injury risk in female collegiate athletes. $N \mathrm{Am} \mathrm{J}$ Sports Phys Ther. 2010;5:47-54.

\begin{tabular}{l|l|l|l|ll}
\hline $\begin{array}{l}\text { Silva, Bezerra, } \\
\text { Cancela }\end{array}$ & 2021 & VOL.15 & N.2 & https://doi.org/10.20338/bjmb.v15i2.200 & 116 of 117
\end{tabular}




\section{ACKNOWLEDGEMENTS}

The authors would like to thank all participants for their availability in collecting the data.

Citation: Silva B, Bezerra P, Cancela JM. Young adults' physical activity and physical function: 12 months follow up. BJMB. 2021. 15(2): 107-117.

Editors: Dr Fabio Augusto Barbieri - São Paulo State University (UNESP), Bauru, SP, Brazil; Dr José Angelo Barela São Paulo State University (UNESP), Rio Claro, SP, Brazil; Dr Natalia Madalena Rinaldi - Federal University of Espírito Santo (UFES), Vitória, ES, Brazil.

Copyright: @ 2021 Silva, Bezerra and Cancela and BJMB. This is an open-access article distributed under the terms of the Creative Commons Attribution-Non Commercial-No Derivatives 4.0 International License which permits unrestricted use, distribution, and reproduction in any medium, provided the original author and source are credited.

Funding: This research received no external funding.

Competing interests: The authors have declared that no competing interests exist.

DOl: https://doi.org/10.20338/bjmb.v15i2.200 